\title{
A. GUINET
}

\section{Transports industriels routiers, un problème d'affectation avec réemploi sous contraintes}

Revue française d'automatique, d'informatique et de recherche opérationnelle. Recherche opérationnelle, tome 18, nº 4 (1984), p. 353-379.

<http://www.numdam.org/item?id=RO_1984_18_4_353_0>

(C) AFCET, 1984, tous droits réservés.

L'accès aux archives de la revue « Revue française d'automatique, d'informatique et de recherche opérationnelle. Recherche opérationnelle » implique l'accord avec les conditions générales d'utilisation (http://www.numdam.org/ legal.php). Toute utilisation commerciale ou impression systématique est constitutive d'une infraction pénale. Toute copie ou impression de ce fichier doit contenir la présente mention de copyright.

\section{Numdam}

Article numérisé dans le cadre du programme

Numérisation de documents anciens mathématiques

http://www.numdam.org/ 


\title{
TRANSPORTS INDUSTRIELS ROUTIERS, UN PROBLĖME D'AFFECTATION AVEC RÉEMPLOI SOUS CONTRAINTES (*)
}

\author{
par A. GUINET $\left({ }^{1}\right)$
}

Résumé. - Plusieurs centaines de transporteurs français de biens industriels recherchent à Theure actuelle une meilleure utilisation de leurs parcs de véhicules, afin de minimiser les problèmes énergétiques qu'ils doivent affronter.

Offrant leurs services à la demande et celle-ci étant entièrement aléatoire, l'organisation de tournées qui minimise la distance parcourue par les véhicules s'effectue mal, et les affectations des moyens de transport aux demandes s'avèrent un délicat problème.

Inspiré de la méthode "Hongroise", un algorithme d'affectation autorisant le réemploi de ressources sous contraintes permet de résoudre ces problèmes, et constitue pour chaque véhicule une tournée en fonction des demandes tout en minimisant son coût d'utilisation.

Cet article présente les principes de cet algorithme et les détails de son implémentation, précédés d'une brève revue des systèmes informatiques de gestion des parcs de véhicules existant à l'heure actuelle ainsi que d'une analyse du problème.

Mots clés : Problèmes de transport; Problème d'affectation avec réemploi; Problème de tournées.

Abstract. - Nowadays, several industrial french carriers search for a better use of their vehicle parks in order to reduce the energy crisis problems which have to face.

The tour organization which minimizes the distance and the number of vehicles required is badly executed, and the vehicle dispatch is a hard problem, because the customer demands are irregular in time and space.

Based on the "Hungarian Method" of $\mathrm{H}$. W. Kuhn, an assignment algorithm allowing the use of the same resource several times solves these problems and finds the tour of each vehicle.

This paper examines the principles of this algorithm and its implementation, after a survey of existing systems about vehicle dispatch in transport and the analysis of the industrial road transport problem.

Keywords: Transportation routing; Assignment routing problem; Delivery problem.

(*) Reçu août 1982.

(1) Laboratoire d'Informatique et d'Automatique Appliquées, M.I.A.G., Université Claude Bernard Lyon I, 43, boulevard du 11-novembre-1918, 69622 Villeurbanne Cedex, France.

R.A.I.R.O. Recherche opérationnelle/Operations Research, 0399-0559/84/04 353 27/\$ 4.70

(C) AFCET-Bordas-Dunod 


\section{INTRODUCTION}

La première partie de cet article dressera un bref panorama des systèmes informatiques de gestion des parcs de véhicules existant dans le domaine des transports. Ceci permettra de préciser les particularités des différents moyens de transport, et distinguera pour chacun d'eux les techniques de résolution employées.

La deuxième partie portera sur le problème plus particulier des transports industriels routiers où peu de choses ont été entreprises à ce jour. Nous poserons le problème de gestion des parcs de véhicules, et nous examinerons ses particularités.

La troisième partie présentera un algorithme d'affectation permettant le réemploi de ressources sous contraintes, qui a été développé pour résoudre les problèmes occasionnés par l'utilisation d'une flotte de véhicules d'un transporteur routier, et justifiera son emploi.

Pour finir, nous préciserons dans la quatrième partie l'implémentation de l'algorithme et les résultats qu'il a permis d'obtenir.

Nous pourrons examiner en annexe l'application de cet algorithme sur un cas posé par une société du sud-est de la France.

\section{PANORAMA DES REALISATIONS INFORMATIQUES ENTREPRISES DANS LE} DOMAINE DES TRANSPORTS

De très grandes disproportions existent entre les différents moyens de transport, dues principalement aux situations économiques très diverses des transporteurs.

L'étude portera successivement sur les transports aériens, urbains, de collectivités, ferroviaires et routiers.

\subsection{Les transports aériens}

Le domaine des transports aériens constitue sans nul doute avec le domaine des transports urbains, le secteur où les études pour l'élaboration de systèmes automatiques de gestion des parcs de véhicules sont les plus nombreuses. $\mathrm{Ce}$ fait s'explique par la situation dans laquelle se trouve le transporteur sur son marché, situation qui est presque toujours celle d'un oligopole ou d'un quasi-monopole.

Ces sociétés de transport publiques ou privées disposant de capacités d'investissement importantes ont pu financer des études informatiques qui ont débouchées sur de nombreux systèmes opérationnels [2,21, 22]. 
Les problèmes de planification de flotte aérienne ne sont pas en général trop contraints. En effet la demande définissant des lignes à desservir régulièrement et s'avérant en outre assez stable, la gestion des parcs de véhicules revient à déterminer principalement le nombre d'avions à affecter aux lignes desservies, en fonction de l'importance de ces dernières au niveau trafic. L'objectif recherché est la réduction des coûts énergétiques des avions.

Les demandes de transport provenant des clients n'indiçant pas les variables des équations mais définissant les constantes de contraintes à vérifier, le problème peut fréquemment se modéliser sous la forme d'un problème d'affectation généralisée pour lequel les algorithmes de programmation linéaire en nombres entiers sont tout à fait indiqués [14]. Il convient de préciser que l'emploi de ces algorithmes s'accompagne souvent de techniques de partitionnement ou d'agrégation de données $[2,22]$.

\subsection{Les transports urbains}

Comme nous l'avons signalé précédemment, de nombreuses réalisations ont vu le jour dans ce domaine.

Ces sociétés de transport définissent dans la majeure partie des cas des monopoles urbains, et bénéficient à ce titre d'une absence presque totale de concurrence.

Leurs possibilités de financement sont importantes, et proviennent soit de leurs fonds propres soit de subventions du contribuable accordées au titre d'aide au service public.

Dans ce domaine les demandes de transport apparaissent beaucoup plus irrégulières et plus fréquentes, mais les lignes à desservir restent toujours stables bien que plus complexes. Les besoins des clients variant tout au long de la journée, si l'on prend cette dernière comme période d'étude nous devons fixer le nombre de véhicules circulant sur les lignes heure par heure, ainsi que la périodicité de ces derniers. Cette différence avec les transports aériens contraint considérablement les problèmes à résoudre, et accroît la taille des données à prendre en compte. L'objectif à atteindre est ici outre la minimisation des coûts énergétiques, la qualité du service à rendre aux usagers.

La programmation linéaire ne pouvant raisonnablement être employée en raison de la complexité des problèmes à résoudre, les outils basés sur l'interactivité homme-machine utilisant des techniques de simulation ainsi que des heuristiques apparaissent tout à fait requis, et l'acquisition de solutions proches de l'optimum de gestion des parcs de véhicules satisfaisante [17, 12, $13,29,16]$. 
Remarquons qu'outre l'utilisation de ces outils pour gérer les réseaux de bus ou de métro, ceux-ci sont largement employés dans le domaine des transports de passagers si l'on veut assurer un service à la clientèle de haute qualité tel le service à la demande $[12,13]$.

\subsection{Les transports de collectivités}

Dans le domaine des transports de collectivités en zone urbaine ou extraurbaine, les études apparaissent nombreuses et ont très souvent été entreprises à la demande de groupements industriels ou d'administrations.

Ces types de transport (ramassage scolaire, acheminement des ouvriers d'une usine) ayant été conçus sur mesure pour satisfaire des besoins particuliers, sont exempts de concurrence et disposent de sources de financement importantes fonction des organismes demandeurs.

Les demandes dans les transports tel le ramassage scolaire sont par définition fixes dans le temps et dans l'espace, en raison de la nature des besoins ayant nécessité celles-cii (ici édıcation). Elles s'avèrent par contre très souvent dispersées.

La gestion des parcs de véhicules revient alors à déterminer un plan de desserte des usagers sur une longue période (par exemple l'année), qui établiera les lignes à parcourir et les horaires de ramassage.

L'objectif est l'octroi d'une qualité de service au moindre coût.

Les outils employés dans ce domaine sont principalement des heuristiques basées sur des techniques d'Analyse Structurale utilisant pour résoudre certains problèmes des algorithmes de programmation linéaire $[18,19,11,3]$.

\subsection{Les transports ferroviaires}

Pour la France les études réalisées par la S.N.C.F. monopole d'état, ont été nombreuses malgré une faible publication de leurs travaux.

N'ayant comme concurrent direct qu'une myriade de transporteurs routiers de petite taille qui se trouvent dispersés sur tout le territoire national, et disposant d'un réseau recouvrant la totalité du pays, les chemins de fer dominent le marché des transports de passagers et de marchandises en zone extra-urbaine.

Les investissements en matériel et logiciel informatiques qu'ils ont effectués jusqu'à présent, ont été nombreux et commandés principalement par leur taille. 
La différence fondamentale de ce moyen de transport vis-à-vis des précédents en ce qui concerne l'utilisation des parcs de véhicules, est la non-stabilité des chemins à laquelle il doit faire face due à la nature aléatoire au niveau géographique des demandes clients. En effet il s'avère difficile de prévoir ces dernières qui peuvent provenir de tout point du territoire national, et bien qu'il existe des lignes préétablies pour satisfaire le marché celles-ci sont souvent insuffisantes.

En plus de la détermination du nombre de trains à accorder aux lignes de desserte pour le trafic de base, le problème posé définit la recherche de l'affectation de moyens de transport supplémentaires aux demandes clients quand celles-ci le nécessitent. L'objectif à atteindre est la minimisation du nombre de locomotives employées et de leur coût d'utilisation.

Les problèmes à résoudre étant toujours des problèmes d'affectation assez contraints, les outils retenus sont principalement des heuristiques $[1,24,6]$.

\subsection{Les transports routiers}

En ce qui concerne les transports routiers, les réalisations entreprises sont peu nombreuses principalement en France, du fait d'un marché composé d'une multitude de sociétés de petite taille disposant de capacités d'investissement très limitées et consacrées avant tout au matériel roulant.

Ce domaine connaît une très vive rivalité interne et subit en outre la lourde concurrence du chemin de fer, aussi bien au niveau du transport de passagers que de biens industriels.

Les problèmes occasionnés par l'utilisation de leurs parcs de véhicules sont complexes, et similaires à ceux du transport ferroviaire étant donnée leur présence sur un même marché.

Les demandes clients en particulier pour le transport de marchandises sont entièrement aléatoires, aussi bien au niveau géographique que temporel. Elles comportent un point de chargement et de livraison distincts du lieu d'implantation du parc de véhicules. La non-stabilité des chemins à parcourir est totale. Le problème généré par le caractère des demandes supplémentaires de la S.N.C.F. se trouve ici généralisé à l'ensemble des demandes.

La gestion des moyens de transport revient à établir les tournées de ces derniers en fonction des besoins de la clientèle de manière à minimiser le nombre de kilomètres parcourus ainsi que le nombre de véhicules utilisés $[9,27]$.

Les outils proposés pour résoudre de tels problèmes sont principalement des heuristiques $[10,8,28,26,25]$, mais il convient de signaler l'emploi 
d'algorithmes de programmation linéaire en nombres entiers dans certains cas $[11,7,23]$.

Les transports maritimes n'ont pas été cités dans ce panorama des moyens de transport, mais on pourrait les rattacher aux problèmes aériens avec lesquels ils partagent de nombreux points communs [5].

\section{LE TRANSPORT INDUSTRIEL ROUTIER}

Plusieurs centaines de transporteurs routiers français disposant de matériel informatique, recherchent à l'heure actuelle un système de gestion de leurs parcs de véhicules, afin de minimiser un problème primordial : les dépenses énergétiques.

Travaillant à la demande, ils souhaiteraient organiser des tournées de véhicules qui leur permettent de satisfaire leur clientèle au plus bas coût.

Dans les paragraphes qui vont suivre, nous allons essayer de dégager et de formuler le problème de ces sociétés.

\subsection{Position du problème}

La gestion d'un parc de véhicules se définit comme la recherche des meilleures affectations, entre les véhicules constituant les ressources et les demandes de transport provenant des clients. L'objectif est de minimiser les coûts issus de l'utilisation de ces véhicules, c'est-à-dire de minimiser le trajet parcouru, les voyages à vide ou non pleins, le nombre de camions utilisés [9].

Les véhicules possèdent le plus souvent les caractéristiques suivantes :

- un volume maximal de marchandises pouvant être transporté;

- un poids maximal de marchandises pouvant être transporté;

- un point de localisation;

- un temps maximal d'utilisation;

- un coût d'utilisation [25].

Ces paramètres sont bien sûr les plus importants, mais on peut en citer d'autres tels que :

- les possibilités de chargement du véhicule;

- les dimensions du véhicule;

- les interdictions d'emploi du véhicule dans certains pays suite à des infractions de la législation routière; 
Entre autres caractéristiques, le type de marchandises pouvant être transporté apparaît plus particulier, car il permet bien souvent une segmentation du problème.

En effet dans la plupart des cas, le problème de transport peut être subdivisé en plusieurs sous-problèmes suivant le type de marchandises, et chacun d'eux donne ainsi lieu à une résolution indépendante des autres.

Au niveau des demandes de transport on note dans la majeure partie des cas les informations suivantes $[9,25]$ :

- un volume de marchandises à transporter;

- un poids de marchandises à transporter;

- un point de chargement des marchandises;

- un point de livraison des marchandises;

- un délai d'exécution du transport.

Il convient de signaler que peu d'articles mentionnent le fait que des demandes de transport peuvent avoir un point de chargement et de livraison distincts du lieu de dépôt du parc de véhicules [7, 11].

On remarque en ce qui concerne le délai d'exécution, que la plupart des commandes sont connues dans la réalité 24 heures avant la date de réalisation au plus tard.

D'autres données apparaissent aussi :

- des facilités de chargement de la marchandise;

- des facilités de déchargement de la marchandise;

- des facilités de circulation sur les points de chargement et de livraison;

- une vitesse moyenne de marche sur le parcours à emprunter en fonction du réseau routier;

- des obligations administratives dues à la législation des douanes;

$-\ldots$

Le type de marchandises là aussi permet un éclatement du problème.

\subsection{Spécificité du problème}

Un certain nombre de faits spécifiques au problème de transport vont conditionner l'outil de résolution recherché.

- L'acquisition de l'information relative aux demandes de transport appartient au domaine du très court terme, et le calcul de prévisions sur ces dernières n'est pas envisageable en raison des caractéristiques de localisation qu'elles comportent.

vol. $18, n^{\circ} 4$, novembre 1984 
- Le problème de transport peut se diviser en plusieurs sous-problèmes indépendants suivant le critère conjoint "type de marchandises" des demandes et des véhicules. On trouvera les produits alimentaires, les produits industriels, les matières dangereuses, etc., et d'autre part les produits à l'exportation, les transports locaux, ...

- Les volumes de marchandises transportés étant standardisés et exprimés en unités connues (palettes, containers, ...), des regroupements de demandes de transport suivant leur localisation s'avèrent envisageables, lorsque celles-ci nécessitent moins d'un véhicule pour leur prise en charge.

- Les demandes de transport une fois regroupées de manière à déterminer un volume et un poids approchant ceux d'un véhicule type, notre problème peut se traduire sous la forme d'un problème d'affectation. (Nous le démontrerons dans la troisième partie, citons [26], [24].)

Il convient de répartir des véhicules entre les demandes de transport en engendrant un coût minimal.

Ce fait est permis par les caractéristiques de poids et de volume qui s'avèrent très souvent identiques pour les véhicules d'un même type de marchandises.

L'optimum d'utilisation d'un parc de véhicules ne correspond pas forcément à la solution proposée par un algorithme d'affectation. En effet cette solution crée des voyages à vide, car un véhicule une fois affecté à une demande l'effectue à partir du dépôt et réintègre celui-ci pour ne plus en bouger.

L'élaboration de tournées peut paraître une solution bien plus judicieuse et proche de notre objectif. Cette notion connue en Recherche Opérationnelle sous le vocable "voyageur de commerce ", doit être incluse à notre méthode d'affectation si l'on veut définir un outil efficace [7, 24].

\section{UN ALGORITHME D'AFFECTATION AVEC REEMPLOI SOUS CONTRAINTES}

\subsection{Notion d'affectation avec réemploi}

G. Monty a développé avec le concours de la Société d'Économie et de Mathématiques Appliquées pour les Études Générales de la S.N.C.F., un algorithme d'affectation avec réemploi permettant la répartition de ressources (locomotives) entre les demandes de transport (trains), chaque ressource pouvant être réemployée sur la période d'étude considérée [24]. Cet algorithme issu de la méthode " Hongroise " de H. W. Kuhn [20], basé sur l'exploitation d'un graphe biparti comportant un ensemble de sommets représentant les 
demandes et un ensemble de sommets représentant les ressources ainsi que leurs réemplois, recherche un couplage maximal de coût minimal entre ces deux ensembles.

Un tel outil qui associe les notions d'affectation et de constitution de tournées par le biais des réemplois, pourrait répondre à notre problème, s'il limitait les affectations de ressources aux différentes contraintes auxquelles sont assujettis les véhicules d'un transporteur routier. Pour les transports ferroviaires il convenait simplement de déterminer sur la période d'étude considérée, le cheminement des locomotives qui devait s'effectuer pour satisfaire le remorquage des trains.

Pour les transports routiers le problème est différent. Le couple " homme-machine " qui constitue la ressource et qui ne peut être dissocié, impose entre autres l'obligation du retour au parc de stationnement après un temps de voyage défini. Notre cheminement s'avère donc contraint par ce nouveau point qui n'était pas pris en compte précédemment.

Le travail entrepris a eu pour but d'adapter l'algorithme de G. Monty aux problèmes du transport par route, en insérant le respect de contraintes supplémentaires relatives à l'utilisation des ressources.

Les paragraphes qui vont suivre montreront que la gestion d'un parc de véhicules routiers peut s'exprimer sous la forme d'un problème d'affectation avec réemploi sous contraintes, et présenteront l'heuristique élaborée.

\subsection{Modélisation du problème de transport routier}

Fonction des décisions énoncées précédemment :

- le modèle représente les demandes de transport et les véhicules relatifs à un type de marchandises donné;

- la période d'étude considérée est du domaine du très court terme (entre 1 et 3 jours);

Soit :

- $I$ l'ensemble des cargaisons $i$ (demandes de transport une fois regroupées), Cardinal $(I)=n$;

- $J^{\prime}$ l'ensemble des véhicules $j^{\prime}$, Cardinal $\left(J^{\prime}\right)=m$;

- $J^{\prime \prime}$ l'ensemble des réemplois des véhicules $j^{\prime \prime}$, Cardinal $\left(J^{\prime \prime}\right)=n$ (un réemploi de ressource est permis lorsqu'une ressource a été affectée à une cargaison, il se trouve donc généré par la satisfaction de la cargaison. Il existe $n$ cargaisons donc $n$ réemplois possibles);

- $J=J^{\prime} \cup J^{\prime \prime}$ l'ensemble des ressources, Cardinal $(J)=m+n$; 
$-c(i, j)$ le coût d'utilisation de la ressource $j$ pour transporter la cargaison $i$, cette valeur est entière;

- $d\left(i_{k}, i_{l}\right)$ le temps de parcours du point de livraison de la cargaison $i_{k}$, au point de livraison de la cargaison $i_{t}(o$ désigne le dépôt);

$-x(i, j)$ cette variable bivalente $=1$ si la ressource $j$ a été affectée à la cargaison $i$ sinon $=0$;

- $t\left(j^{\prime}\right)$ le temps de disponibilité du véhicule $j^{\prime}$;

$-w(j)$ cette variable spécifie l'indice du véhicule auquel se rapporte la ressource $j$ après affectation.

Contraintes d'affectation des ressources:

$$
\sum_{i=1}^{n} x(i, j) \leqslant 1, \quad \forall j=1, \ldots, m+n .
$$

Contraintes d'affectation des cargaisons :

$$
\sum_{j=1}^{m+n} x(i, j)=1, \quad \forall i=1, \ldots, n
$$

Variables bivalentes :

$$
x(i, j)=0 \text { ou } 1, \quad \forall i=1, \ldots, n, \quad \forall j=1, \ldots, m+n .
$$

Contraintes de réemploi :

Les $m$ premières ressources de $J$ représentent les véhicules, les $n$ dernières ressources de $J$ définissent les réemplois des véhicules. La ressource de rang $m+i$ est le réemploi de véhicule généré par la satisfaction de la cargaison $i$ :

$$
\left.\begin{array}{c}
x(i, m+i)=0, \quad \forall i=1, \ldots, n, \\
\mathrm{w}(\mathrm{j})=\sum_{i=1}^{n}(x(i, j) * j), \quad \forall j=1, \ldots, m, \\
w(m+i)=\sum_{k=1}^{n}\left(x(k, m+i) *\left(\sum_{j=1}^{m+n}(x(i, j) * w(j))\right)\right), \\
\forall i \in I, \\
\sum_{i=1}^{n} x(i, j) \leqslant w(j), \quad \forall j=m+1, \ldots, m+n .
\end{array}\right\}
$$


Contraintes de disponibilité des véhicules :

$$
\begin{aligned}
& \sum_{i=1}^{n}(x(i, j) *(d(o, i)+d(i, o))) \\
& +\sum_{\substack{r \forall x=1, \ldots, n ; \\
w(m+k)=j]}} \sum_{l=1}^{n}(x(l, m+k) \\
& \text { * }(d(k, l)+d(l, o)-d(k, o))) \leqslant t(j) \text {, } \\
& \forall j=1, \ldots, m \text {. }
\end{aligned}
$$

Fonction objectif :

$$
\text { MINIMISER } z=\sum_{i=1}^{n} \sum_{j=1}^{m+n}(x(i, j) * c(i, j)) \text {. }
$$

Il convient à la suite de cette modélisation qui a principalement un intérêt descriptif, de souligner quelques points importants.

- Lorsqu'on value $m$ à 1 , notre modèle représente le problème de l'affectation d'un véhicule unique entre plusieurs cargaisons. Le principe du réemploi permettant au véhicule de transporter l'ensemble des cargaisons en série, une solution peut être trouvée dans la mesure où la contrainte de disponibilité du véhicule [équation (6)] l'autorise. Les variables $w(j)$ précisent dans ce cas que toute ressource employée pour transporter une cargaison [équation (5)] est à rattacher au véhicule puisque $w(j)=1, \forall j=1, \ldots, m$. Ce problème de transport définit un problème de recherche de chemin hamiltonien de coût minimal. Les sommets du graphe représentant les cargaisons et les coûts d'affectation valuant chaque arc, le trajet du véhicule passera une fois et une fois seulement par chacun des sommets du graphe puisque le véhicule doit transporter chaque cargaison et ne peut transporter deux fois la même cargaison [équation (2)].

- En outre si les coûts d'affectation $c(i, j)$ sont égaux :

- pour les véhicules, à la distance du dépôt au point de chargement de la cargaison $i$, plus la distance du point de chargement au point de livraison de la cargaison $i$, plus la distance du point de livraison de la cargaison $i$ au dépôt;

- pour les réemplois de véhicules, à la distance du point de livraison de la cargaison $j-m$ créant le réemploi au point de chargement de la cargaison $i$, plus la distance du point de chargement au point de livraison de la cargaison $i$, plus la distance du point de livraison de la cargaison $i$ au dépôt, moins la distance du point de livraison de la cargaison $j-m$ au dépôt; 
notre chemin hamiltonien de coût minimal représente avec l'adjonction du dépôt un circuit hamiltonien de coût minimal, solution de problèmes du type " voyageur de commerce".

- Notre problème est donc $N P$ complet puisqu'il correspond à un problème de voyageurs de commerce multiples.

\subsection{Heuristique de résolution proposée}

On recherche un couplage maximal de coût minimal entre un ensemble de cargaisons et un ensemble de ressources.

A cette fin, nous avons divisé notre problème d'affectation avec réemploi en deux problèmes distincts, un problème principal portant sur l'étude du problème dual du problème d'affectation relaxé des contraintes de réemploi et de disponibilité des véhicules [équations (4), (5) et (6)], et un sous-problème portant sur la recherche d'un couplage de cardinalité maximale dans un graphe orienté biparti initialisé à partir d'une solution issue de l'étude du problème principal.

DÉFINITION : Soit un graphe biparti orienté $G(X, Y, U)$ :

- $X$ étant l'ensemble des sommets représentant les cargaisons $x_{i}, i$ appartenant à $I$;

- $Y$ étant l'ensemble des sommets incluant l'ensemble $Y^{\prime}$ des véhicules $y_{j}$, $j$ appartenant à $J^{\prime}$, et l'ensemble $Y^{\prime \prime}$ des réemplois $y_{i}, i$ appartenant à $I$ (réemploi de la ressource préalablement affectée à la cargaison $i$, quelle que soit la ressource);

- $U$ étant un ensemble d'arcs représentant entre autres les possibilités d'affectation, tel qu'aucun arc n'ait ses deux extrémités situées dans $X$ ou dans $Y$.

L'ensemble $U$ peut contenir trois types d'arcs :

- des arcs $u$ qui ont pour extrémité initiale un sommet $x$ appartenant à $X$ et pour extrémité terminale un sommet $y$ appartenant à $Y$, ils représentent les possibilités d'affectation non retenues par la solution en cours du sousproblème;

- des arcs $w$ qui ont pour extrémité initiale un sommet $y$ appartenant à $Y$ et pour extrémité terminale un sommet $x$ appartenant à $X$, ils représentent les possibilités d'affectation retenues par la solution en cours du sousproblème;

- des arcs $v$ qui ont pour extrémité initiale un sommet $y$ appartenant à $Y^{\prime \prime}$ et pour extrémité terminale un sommet $x$ appartenant à $X$, ils précisent l'origine d'un réemploi de ressource. 
Un couplage est un ensemble d'arcs $u$ appartenant à $U$ notés $w$, vérifiant pour les sommets $y$ appartenant à $Y^{\prime \prime}$ les contraintes de réemploi leur étant associées [équations (4) et (5)], vérifiant pour tout sommet $y$ appartenant à $Y$ les contraintes de disponibilité des véhicules [équation (6)], tel qu'aucun couple d'arcs ne possède des extrémités initiales communes ou des extrémités terminales communes. Le couplage définit la solution du sous-problème.

Les sommets des arcs $w$ sont dits saturés.

Une chaîne alternée est une chaîne d'arcs appartenant à $U$. Une chaîne alternée augmentante est une chaîne alternée sans cycle dont l'extrémité initiale est un sommet $x$ appartenant à $X$ non saturé, et dont l'extrémité terminale est un sommet $y$ appartenant à $Y$ non saturé. Dans le cas où l'extrémité terminale de la chaîne est un sommet $y$ appartenant à $Y^{\prime \prime}$ la contrainte de réemploi associée au sommet $y$ doit en outre être vérifiée [équations (4) et (5)].

Un transfert est une opération consistant à partir de l'existence d'une chaîne alternée augmentante de changer les arcs $u$ de la chaîne alternée augmentante en arcs $w$, de changer les arcs $w$ d'origine de la chaîne alternée augmentante en $\operatorname{arcs} u$, et de supprimer les arcs $v$ de la chaîne alternée augmentante ainsi que l'éventuel arc $v$ dont l'extrémité terminale est l'extrémité initiale de la chaîne.

Nous prouvons [15] qu'un transfert permet de passer d'un couplage donné à un nouveau couplage de cardinalité supérieure, et qu'à partir d'un couplage donné lorsque plus aucun transfert n'est possible le couplage est de cardinalité maximale, dans le cas où le problème posé admet une solution.

\section{Algorithme :}

PROCEDURE PRINCIPALE

*/ Recherche d'une solution de base du problème dual du problème d'affectation relaxé des contraintes de réemploi et de disponibilité des véhicules, et définition du sous-problème à partir de la solution duale $/^{*}$

APPEL PROCEDURE initialisation

*/ Recherche d'une solution duale vérifiant l'ensemble des contraintes, c'est-à-dire d'un couplage de $G$ solution du sous-problème, de cardinalité égale à $n / *$

TANT QUE la cardinalité du couplage de $G<n$ FAIRE

*/ Recherche d'un couplage de $G$ de cardinalité maximale $/ *$

APPEL PROCEDURE couplage

SI la cardinalité du couplage de $G<n$

ALORS

*/ La solution du sous-problème ne vérifie pas toutes les contraintes [équation (2)], on recherche une autre solution du problème dual $/^{*}$

APPEL PROCEDURE extension

FINSI

FINFAIRE

FIN PROCEDURE

vol. $18, \mathrm{n}^{\circ} 4$, novembre 1984 
Initialisation du graphe $G$.

Soit le problème dual du problème d'affectation avec réemploi relaxé des contraintes de réemploi et de disponibilité des véhicules.

D

$$
\text { MINIMISER } w=\sum_{i=1}^{n} u(i)+\sum_{j=1}^{n+m} v(j)
$$

sous les contraintes :

$$
\begin{gathered}
u(i)+v(j) \geqslant-c(i, j), \quad \forall i=1, \ldots, n, \quad \forall j=1, \ldots, m+n, \\
u(i) \text { de signe quelconque, } \forall i=1, \ldots, n, \\
v(j) \geqslant 0, \quad \forall j=1, \ldots, m+n .
\end{gathered}
$$

Une solution de base du problème $D$ peut être :

$$
\begin{gathered}
u(i)=\underset{\forall j \in J}{\operatorname{MAXIMUM}}(-c(i, j))=-\underset{\forall j \in J}{\operatorname{MINIMUM}}(c(i, j)), \\
\forall i=1, \ldots, n, \\
v(j)=0, \quad \forall j=1, \ldots, m+n
\end{gathered}
$$

PROCEDURE initialisation

CALCULER matrice $C$

*/ Chaque coût $C(i, j)$ est calculé en fonction de la localisation de la ressource $j$, du point de chargement et de livraison de la cargaison $i$, et éventuellement de la situation géographique du dépôt $/ *$

* Définition des arcs du graphe $G$ en fonction de la solution de base du problème dual. Il existe un arc $u$ entre $i$ et $j$ si et seulement si $u(i)+v(j)=-c(i, j)$ ou encore $c(i, j)+u(i)+v(j)=0$. A ce niveau $c(i, j)=C(i, j) /^{*}$

POUR $i=1$ à $n$

FAIRE

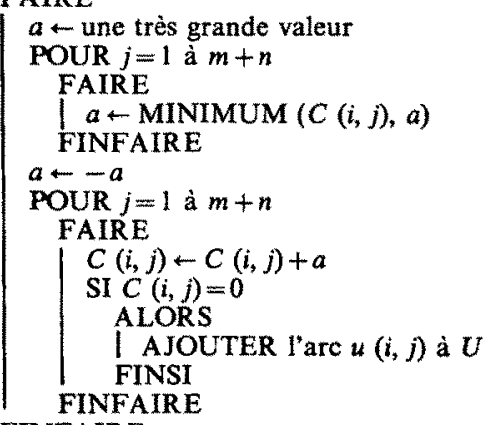

\section{FINFAIRE}

AJOUTER les arcs $v$ à $U$

*/ On inclut dans le graphe $G$ l'ensemble des arcs $v$ précisant l'origine des réemplois de ressources sommets du graphe $G / *$

FIN PROCEDURE

Recherche d'un couplage de cardinalité maximale.

Afin de résoudre notre sous-problème, on recherche dans le graphe $G$ représentant une solution duale, un couplage de cardinalité maximale. $\mathrm{Ce}$

R.A.I.R.O. Recherche opérationnelle/Operations Research 
couplage ne pourra avoir une cardinalité supérieure à $n$, et solutionnera notre problème lorsqu'il aura une cardinalité égale à $n$.

Les arcs $u$ de $G$ matérialisant les possibilités d'affectation, il convient de sélectionner pour la constitution du couplage, uniquement ceux dont les sommets $y$ appartenant à $Y^{\prime}$ vérifient les contraintes de disponibilité des véhicules et ceux dont les sommets $y$ appartenant à $Y^{\prime \prime}$ vérifient les contraintes de réemploi ainsi que les contraintes de disponibilité des véhicules.

Si un couplage de cardinalité égale à $n$ est trouvé, il définit la solution de notre problème et notre étude est terminée, sinon il convient de trouver une autre solution duale qui nous permettra de redéfinir notre graphe $G$ en cherchant à le compléter.

PROCEDURE couplage

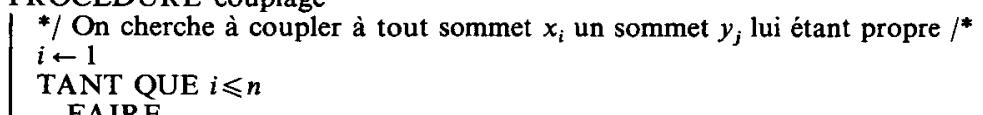

FAIRE

SI $x_{i}$ non saturé

ALORS

*/ On cherche en parcourant le graphe $G$ une chaîne alternée augmentante d'extrémité initiale $x_{i}$. Cette chaîne de longueur impaire peut être constituée d'un arc $u$, d'un triplet d'arcs $u, v, u$, d'un triplet d'arcs $u, w, u, \ldots /^{*}$

MASQUER l'arc $v$ d'extrémité terminale $x_{i}$ s'il existe

*/ On cherche tout d'abord une chaîne alternée augmentante de longueur 1 d'extrémité initiale $x_{i} / *$

CHERCHER un arc $u$ d'extrémité initiale $x_{i}$ et d'extrémité terminale $y_{j}, y_{j}$ étant non saturé et vérifiant la contrainte de réemploi si il appartient à $Y^{\prime \prime}$.

*/ Dans le cas où il n'existe pas de chaîne alternée augmentante de longueur 1 d'extrémité initiale $x_{i}$, on recherche une chaîne alternée augmentante de longueur supérieure à 1 d'extrémité initiale $x_{i} / *$

SI il n'existe pas une chaîne alternée augmentante de longueur 1 d'extrémité initiale $x_{i}$

ALORS

I APPEL PROCEDURE chaîne

FINSI

TANT QU'il existe une chaîne alternée augmentante non étudiée ET qu'aucune chaîne alternée augmentante vérifiant les contraintes de disponibilité des véhicules n'a été trouvée

FAIRE

*/ On vérifie que la chaîne alternée augmentante satisfait les contraintes de disponibilité des véhicules [équation (6)] /*

APPEL PROCEDURE disponibilité

SI la chaîne alternée augmentante ne vérifie pas les contraintes de disponibilité ALORS

APPEL PROCEDURE chaîne

FINSI

FINFAIRE

DEMASQUER l'arc $v$ d'extrémité terminale $x_{i}$ s'il existe

SL Lune chaîne alternée augmentante vérifiant les contraintes de disponibilité des véhicules existe

\section{ALORS}

APPEL rROCEDURE transfert

FINSI

FINSI

$i \leftarrow i+1$

FINFAIRE

FIN PROCEDURE 
PROCEDURE disponibilité

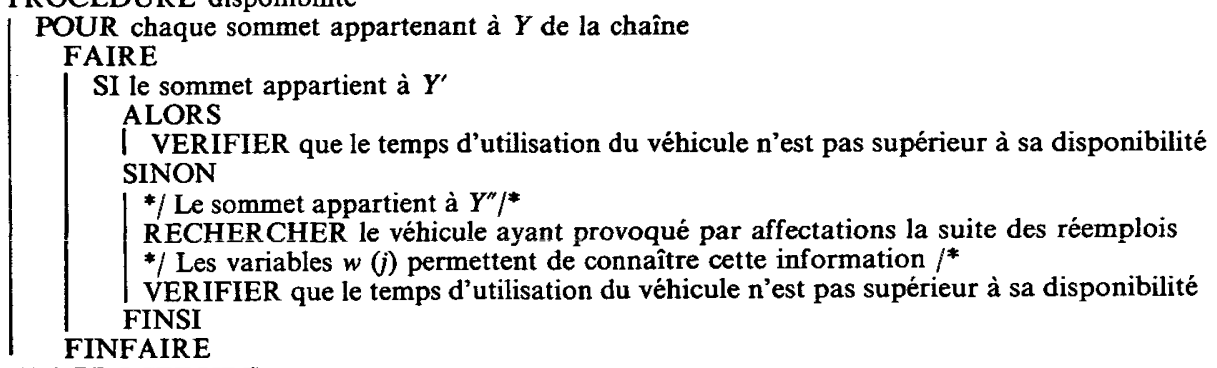

FIN PROCEDURE

\section{PROCEDURE transfert}

*/ On effectue un transfert pour augmenter le couplage $/^{*}$

SUPPRIMER l'arc $v$ d'extrémité terminale $x_{i}$ s'il existe

SUPPRIMER les arcs $v$ de la chaîne

CHANGER les arcs $u$ de la chaîne en arcs $w$

CHANGER les arcs $w$ d'origine de la chaîne en arcs $u$

*/ Par le biais des réemplois l'accroissement du couplage peut permettre l'existence de nouvelles chaînes alternées augmentantes interdites jusqu'ici par les contraintes de réemploi /*

$i \leftarrow 0$

FIN PROCEDURE

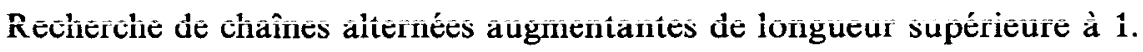

On cherche une chaîne alternée augmentante de longueur supérieure à 1 . La " PROCEDURE chaîne " recherche une chaîne alternée d'extrémité initiale $x_{i}$ et d'extrémité terminale $y_{j}, y_{j}$ étant non saturé et vérifiant la contrainte de réemploi lui étant associée dans le cas où il appartient à $Y^{\prime \prime}$.

La recherche des chaînes alternées augmentantes est effectuée par énumération séquentielle des différentes chaînes du graphe $G$ ayant pour extrémité initiale $x_{i}$, suivant des principes comparables à l'algorithme de construction d'un arbre alterné décrit par Gondran et Minoux [14]. Cette recherche revient à construire un arbre alterné constitué d'arcs $u$ ou $u, v$ ou $u$, w, et à effectuer une exploration en profondeur de cet arbre.

Extension du graphe réduit.

La solution duale n'ayant pu permettre la détermination d'un couplage de cardinalité égale à $n$, il convient de chercher une autre solution duale si possible de valeur moindre. Cette nouvelle solution devra faire apparaitre de nouvelles possibilités d'affectation, c'est-à-dire de nouveaux arcs $u$ dans notre graphe $G$.

Pour cela il convient de marquer pour mémoire les actuelles possibilités d'affectation (extrémités des arcs $u$ ) qui n'ont pas pu permettre de satisfaire le transport de certaines cargaisons, dans le but de porter la recherche de nouvelles possibilités d'affectation entre des cargaisons marquées et des ressources non marquées. Nous marquons tout sommet de toute chaîne alternée issue d'un sommet $x_{i}$ non saturé. 


\section{Définitions :}

- est dit marqué, tout sommet $y_{j}$ extrémité terminale d'une chaîne alternée dont l'extrémité initiale est un sommet $x_{i}$ non saturé;

- est dit marqué, tout sommet $x_{i}$ non saturé, ou tout sommet $x_{i}$ extrémité terminale d'un arc $v$ ou $w$ dont l'extrémité initiale est marquée.

Notre matrice $C$ représente les valeurs $c(i, j)+u(i)+v(j)$. Recherchons $a$ le minimum des $C(i, j)$ pour tout sommet $x_{i}$ marqué et tout sommet $y_{j}$ non marqué $(a>0)$.

Une nouvelle solution de notre problème dual peut être :

$$
\begin{array}{ll}
u(i) \leftarrow u(i)-a, & \forall \text { sommet } x_{i} \text { marqué, } \\
v(j) \leftarrow v(j)+a, & \forall \text { sommet } y_{j} \text { marqué. }
\end{array}
$$

Nous pouvons constater que premièrement nous obtenons bien une solution de notre problème dual et que deuxièmement de nouvelles possibilités d'affectation apparaissent. Plusieurs cas se présentent :

- $i$ et $j$ sont soit tous deux marqués, soit tous deux non marqués, la somme des valeurs des variables duales leur étant associées reste inchangée (les sommets des arcs $w$ étant soit tous deux marqués, soit tous deux non marqués, le couplage est conservé d'une solution duale à l'autre);

- $i$ est marqué, $j$ est non marqué [il n'existe pas de par la définition du marquage d'arc $u$ entre $i$ et $j$, donc $: u(i)+v(j)>-c(i, j)$,

$$
u(i)+v(j)-a \geqslant-c(i, j)
$$

de nouvelles possibilités d'affectation apparaissent dans ce cas;

- $i$ est non marqué, $j$ est marqué [deux cas existent $u(i)+v(j)-c(i, j)$ ou $u(i)+v(j)=-c(i, j)] u(i)+v(j)+a>-c(i, j)$ des anciennes possibilités d'affectation ne vérifiant pas certaines contraintes peuvent disparaître.

Lorsque nous ne tenons pas compte des contraintes de disponibilité des véhicules nous prouvons que la valeur de la solution duale $w$ décroît ou reste stable à chaque changement de solution [15]. Dans le cas contraire nous remarquons seulement qu'elle décroît très souvent.

\section{PROCEDURE extension}

*/ Marquage des sommets du graphe /*

POUR tout sommet $x_{i}$ de $G$ non saturé

FAIRE

POUR toute chaîne alternée d'extrémité initiale $x_{i}$

FAIRE

I MARQUER les sommets de la chaîne FINFAIRE

FINFAIRE

*/ Changement de solution duale $/ *$

$a \leftarrow$ une très grande valeur 
POUR tout $i$ correspondant à un sommet $x_{i}$ marqué

FAIRE

POUR tout $j$ correspondant à un sommet $y_{j}$ non marqué

FAIRE

$a \leftarrow \operatorname{MINIMUM}(C(i, j), a)$

FINFAIRE

FINFAIRE

POUR tout $i$ correspondant à un sommet $x_{i}$ marqué

FAIRE

POUR $j=1$ à $m+n$

FAIRE

I $C(i, j) \leftarrow C(i, j)-a$

FINFAIRE

FINFAIRE

POUR tout $j$ correspondant à un sommet $y_{j}$ marqué

FAIRE

POUR $i=1$ à $n$

FAIRE

I $C(i, j) \leftarrow C(i, j)+a$

FINFAIRE

FINFAIRE

FIN PROCEDURE

\section{Exemple :}

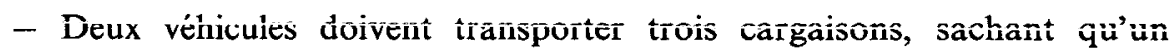
véhicule ne peut prendre en charge les trois cargaisons pour des raisons de temps de chargement et de livraison de la marchandise.

- Soit $C$ :

\begin{tabular}{|c|r|r|r|r|c|}
\hline \multirow{2}{*}{ Cargaisons } & \multicolumn{5}{|c|}{$\begin{array}{c}\text { Véhicules } \\
\text { et réemplois }\end{array}$} \\
\cline { 2 - 6 } & 1 & 2 & 3 & 4 & 5 \\
\hline $1 \ldots \ldots \ldots \ldots \ldots$ & 24 & 25 & $\infty$ & 20 & 12 \\
$2 \ldots \ldots \ldots \ldots \ldots$ & 20 & 13 & 0 & $\infty$ & 10 \\
$3 \ldots \ldots \ldots \ldots$ & 14 & 21 & 8 & 16 & $\infty$ \\
\hline
\end{tabular}

- Initialisation :

\begin{tabular}{|c|c|c|c|c|c|}
\hline$C$ & 1 & 2 & 3 & 4 & 5 \\
\hline 1 & 12 & 13 & $\infty$ & 8 & 0 \\
2 & 20 & 13 & 0 & $\infty$ & 10 \\
3 & 6 & 13 & 0 & 8 & $\infty$ \\
\hline
\end{tabular}

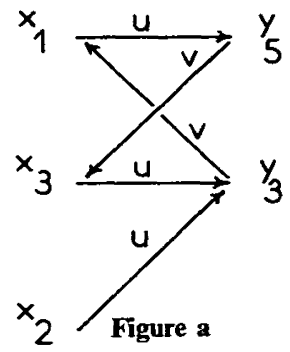

R.A.I.R.O. Recherche opérationnelle/Operations Research 
Couplage ( $\varnothing$ ).

Sommets marqués $\left(x_{1}, x_{2}, x_{3}, y_{3}, y_{5}\right)$.

Valeur de $a$ égale à 6 .

- Première extension du graphe $G$ :

\begin{tabular}{|c|c|c|c|c|c|}
\hline$C$ & 1 & 2 & 3 & 4 & 5 \\
\hline 1 & 6 & 7 & $\infty$ & 2 & 0 \\
2 & 14 & 7 & 0 & $\infty$ & 10 \\
3 & 0 & 7 & 0 & 2 & $\infty$ \\
\hline
\end{tabular}
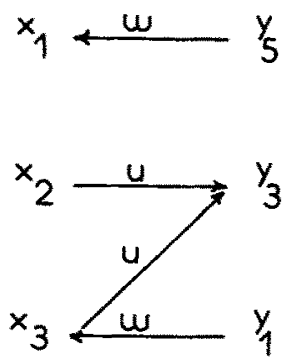

Figure b

Couplage $\left(\left(y_{1}, x_{3}\right),\left(y_{5}, x_{1}\right)\right)$.

L'affectation de $y_{3}$ à $x_{2}$ est interdite par la contrainte de temps.

Sommets marqués $\left(x_{2}, y_{3}\right)$.

Valeurs de $a$ égale à 7 .

- Deuxième extension du graphe $G$ :

\begin{tabular}{|c|c|c|c|c|c|}
\hline$C$ & 1 & 2 & 3 & 4 & 5 \\
\hline 1 & 6 & 7 & $\infty$ & 2 & 0 \\
2 & 7 & 0 & 0 & $\infty$ & 3 \\
3 & 0 & 7 & 7 & 2 & $\infty$ \\
\hline
\end{tabular}
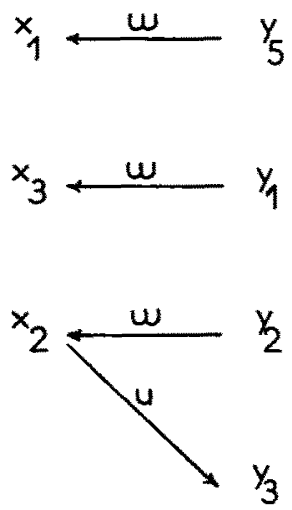

Couplage $\left(\left(y_{1}, x_{3}\right),\left(y_{5}, x_{1}\right),\left(y_{2}, x_{2}\right)\right)$.

Figure c

vol. $18, \mathrm{n}^{\circ} 4$, novembre 1984 
- Le véhicule $\mathrm{n}^{\circ} 1$ transportera les cargaisons $\mathrm{n}^{\text {os }} 3$ et 1 , tandis que le véhicule $\mathrm{n}^{\circ} 2$ transportera la cargaison 2 .

\section{IMPLÉMENTATION DE L'ALGORITHME}

La réalisation de la maquette införmatique traduisant l'algorithme développé dans cet article, a été entreprise en fonction des souhaits du transporteur routier qui a posé initialement le problème, et des possibilités de financement informatique des sociétés de transport disposant de plus de 20 véhicules.

La maquette finale a été prévue pour fonctionner sur un micro-ordinateur organisé autour d'un processeur Z 80 ou 8088 d'Intel, comprenant un disque dur de 10 millions d'octets et utilisant le système d'exploitation $\mathrm{CP} / \mathrm{M}$ ou CP/M86 de Digital Research.

Actuellement le logiciel a été réalisé sur un mini-ordinateur Hewlett Packard modèle 3000 , en raison de la configuration matérielle et des outils de développement qu'offre ce dernier.

La maquette a été écrite en Fortran 4 pour des raisons de portabilité, se compose de 1000 instructions et nécessite une place disponible en mémoire centrale de 40000 octets.

Nous allons tout d'abord examiner les principaux aspects de ce logiciel, et nous verrons dans un deuxième temps les résultats qu'il a permis d'obtenir.

\subsection{Réalisation d'une maquette}

Les principaux objets utilisés par ce logiciel sont les suivants :

- une matrice $C$ contenant les coûts de la réalisation de $n$ cargaisons par $m$ véhicules et $n$ réemplois;

- une matrice $l$ listant tous les arcs du graphe $G$;

- une matrice $a$ contenant l'ensemble des arcs $w$;

- un vecteur ch utilisé pour constituer les chaînes alternées;

- une matrice $t$ des temps de parcours entre les points de livraison des cargaisons;

- un vecteur $d$ des disponibilités horaires des véhicules.

La réalisation du logiciel a donné lieu à l'écriture de plusieurs modules programmes, chacun ayant une fonction propre, afin de pouvoir tester plus facilement chaque grand type d'opération dans notre graphe. 
La procédure principale a pour but :

- l'appel de la procédure d'initialisation des objets et de constitution du graphe $G$ de départ;

- l'appel de la procédure de recherche du couplage maximal;

- l'appel de la procédure d'extension du graphe;

- l'écriture des résultats.

La procédure d'initialisation des objets calcule les données des matrices $C$, $t$ et $d$, à partir d'informations relatives aux cargaisons et aux véhicules. La formule utilisée pour la détermination des coûts s'avère très importante et va conditionner toutes les affectations futures. En effet, certains véhicules se trouvant hors du dépôt au départ du problème, il convient de savoir pour le calcul de $C$, si on doit prendre en compte le fait que tout véhicule doit regagner son lieu de stationnement même s'il n'est pas employé. De même en fin de période, on peut ou non inclure le coût du retour au dépôt pour chaque véhicule ou réemploi.

Pour ce qui concerne le calcul des distances de ville à ville, des coordonnées géographiques ont été attribuées à chaque commune. L'imprécision due à la détermination d'une distance à « vol d'oiseau », a été corrigée par la définition d'un trajet à suivre pour toute cargaison.

Les contraintes relatives aux incompatibilités de certains couples "véhicule-cargaison" qui ont été évoquées dans la deuxième partie, sont prises en compte par l'attribution de coûts infinis.

En outre la procédure calcule la solution de base du problème dual, et elle constitue la matrice $l$ représentant le graphe $G$.

La procédure de recherche du couplage maximal a pour fonction :

- la recherche par lecture séquentielle de la matrice $l$, de la plus petite chaîne joignant un sommet $x$ non saturé à un sommet $y$ non saturé (chaîne comportant un arc $u$ unique);

- l'appel d'une procédure de recherche de chaînes plus grandes;

- la mise à jour des matrices $a$ et $l$.

La procédure de recherche de chaînes supérieures à un arc, permet de déterminer une chaîne joignant un sommet $x$ à un sommet $y$ tous deux insaturés, si cette chaîne existe. Cette procédure aurait pu être programmée de manière récursive si le langage l'avait permis, elle procède par balayages séquentiels de la matrice $l$ et constitue petit à petit le vecteur ch, en gérant 
une pile qui autorise une exploration méthodique de toutes les chaînes d'arcs issues du sommet étudié.

Elle vérifie en outre qu'aucun cycle ne puisse se former entre les réemplois.

La procédure d'extension du graphe a pour but de faire apparaître de nouvelles possibilités d'affectation (arcs $u$ et $v$ ) en calculant une nouvelle solution duale suivant les règles énoncées dans l'algorithme.

La procédure de vérification des disponibilités des véhicules appelée par la procédure de recherche du couplage maximal, contrôle pour chaque sommet $y$ du vecteur ch le respect des disponibilités horaires des véhicules, en fonction des affectations proposées et déjà effectuées.

Le principal problème rencontré lors du développement du logiciel a été de concilier temps de calcul et place en mémoires principale et secondaire.

En outre la détermination d'une formule de calcul des coûts de la matrice $C$, a mise à jour un délicat problème qui dépend de la politique commerciale et sociale du transporteur.

\section{2. Résultats obtenus}

La validation de l'heuristique a été acquise par la qualité des solutions obtenues lors des différents jeux d'essais entrepris sur la maquette, qui sera prochainement complétée et commercialisée par une société de services. En annexe, des données illustrant l'activité moyenne d'un transporteur pour un type de marchandises, ainsi que les résultats obtenus, donnent une idée des possibilités du logiciel. L'aquisition de la solution pour la répartition d'une trentaine de véhicules entre une quarantaine de cargaisons, a nécessité moins de 2 minutes de temps calcul.

A titre purement indicatif, une heuristique permettant l'affectation de locomotives à des trains développée par J. M. P. Boller sur un ICL 1904s, requiert 3 à 10 minutes pour la résolution d'un jeu d'essais comportant 30 à 50 trains [6].

L'algorithme d'affectation avec réemploi sous contraintes a en outre été testé sur d'autres problèmes.

Tout d'abord par le biais de l'attribution de coûts infinis aux réemplois, on peut résoudre de simples problèmes d'affectation, si le nombre de véhicules est supérieur au nombre de cargaisons.

D'autres types de problèmes basés sur la recherche dans un graphe d'un chemin hamiltonien de coût minimal, ont été solutionnés. En effet la réparti- 
tion d'une ressource unique entre plusieurs demandes, résout certains problèmes d'ordonnancement ou des problèmes du type «voyageur de commerce ".

Enfin quelques problèmes de l'Analyse de Données comme la recherche d'une classification à partir d'un indice de distance ou de similitude, tel "l'évolution des vases archaïques chinois" ont été résolus.

\section{REMERCIEMENTS}

Je tiens à remercier Michel Dang pour ses critiques avisées de mes travaux, pour ses conseils et ses encouragements.

\section{ANNEXES}

Ces données ont été gracieusement fournies par une société de transport du sud-est de la France, disposant d'une flotte routière composée de plusieurs centaines de véhicules.

Elles représentent pour une agence de la société pour une période donnée :

- les cargaisons à transporter dans la catégorie «transport industriel sur courte distance ");

- la situation du parc de véhicules de la même catégorie à la veille de cette période.

PARC ROUTIER

\begin{tabular}{|c|cc|c|cr|}
\hline $\begin{array}{c}\text { Numéro } \\
\text { des } \\
\text { véhicules }\end{array}$ & $\begin{array}{c}\text { Point } \\
\text { de stationnement } \\
\text { des véhicules }\end{array}$ & $\begin{array}{c}\text { Numéro } \\
\text { des } \\
\text { véhicules }\end{array}$ & $\begin{array}{c}\text { Point } \\
\text { de stationnement } \\
\text { des véhicules }\end{array}$ \\
\hline 01 à 04 & Marseille & 13 & 18 & Pierrelatte & 26 \\
05 & Lyon & 69 & 19 & Granes (Livron) & 26 \\
06 & Grenoble & 38 & 20 & Les Échelles & 73 \\
07 & Valence & 26 & 21 & Chambery & 73 \\
08 & Saint-Étienne & 42 & 22 & Saint-Priest (Lyon) & 69 \\
09 & Saint-Agrève & 07 & 23 à 24 & Nîmes & 30 \\
10 à 14 & Livron & 26 & 25 & Arles & 13 \\
15 & Loriol & 26 & 26 & Grenoble & 38 \\
16 & Charols (Bourdeaux) & 26 & 27 & Villefort (Privas) & 07 \\
17 & Clermont-Ferrand & 63 & 28 & Aimargues & 30 \\
\hline
\end{tabular}

\section{DIVERS RENSEIGNEMENTS}

Consommation des véhicules :

- 321 pour $100 \mathrm{~km}$ à vide;

- 441 pour $100 \mathrm{~km}$ en pleine charge.

vol. $18, n^{\circ} 4$, novembre 1984 
Vitesse des véhicules :

- une moyenne de $70 \mathrm{~km}$ par heure.

Localisation du dépôt :

- Livron (Drôme, 26).

\section{CARGAISONS}

\begin{tabular}{|c|c|c|c|c|}
\hline $\begin{array}{l}\text { Numéro } \\
\text { de la } \\
\text { cargaison }\end{array}$ & Point de chargement & & Point de livraison & \\
\hline $\begin{array}{l}01 \\
02 \\
03 \\
04 \\
05 \\
06 \\
07 \\
08 \\
09 \\
10 \\
11 \\
12 \\
13 \\
14 \\
15 \\
16 \\
17 \\
18 \\
19 \\
20 \\
21 \\
22 \\
23 \\
24 \\
25 \\
26 \\
27 \\
28 \\
29 \\
30 \\
31 \\
32 \\
33 \\
34 \\
35 \\
36\end{array}$ & $\begin{array}{c}\text { Loriol } \\
\text { Beauchastel (La Voulte) } \\
\text { Grenoble } \\
\text { Valence } \\
\text { Crest } \\
\text { Saulce (Loriol) } \\
\text { Aubenas } \\
\text { Crest } \\
\text { Loriol } \\
\text { Luituoi } \\
\text { Livron } \\
\text { Roanne } \\
\text { Livron } \\
\text { Le Cheylard } \\
\text { Pierrelatte } \\
\text { Livron } \\
\text { Saint-Agrève } \\
\text { Valence } \\
\text { Saint-Fons (Lyon) } \\
\text { Les Échelles } \\
\text { Fos-sur-Mer } \\
\text { Livron } \\
\text { Livron } \\
\text { Livron } \\
\text { Livron } \\
\text { Nîmes } \\
\text { Aindancette (Saint-Donat) } \\
\text { Saint-Agrève } \\
\text { Saint-Agrève } \\
\text { La Mure } \\
\text { Nîmes } \\
\text { Moulins } \\
\text { Loriol } \\
\text { Annonay } \\
\text { Dijon } \\
\text { Saint-Etienne }\end{array}$ & $\begin{array}{l}26 \\
07 \\
38 \\
26 \\
26 \\
26 \\
07 \\
26 \\
26 \\
26 \\
26 \\
42 \\
26 \\
07 \\
26 \\
26 \\
07 \\
26 \\
69 \\
73 \\
13 \\
26 \\
26 \\
26 \\
26 \\
30 \\
26 \\
07 \\
07 \\
38 \\
30 \\
03 \\
26 \\
07 \\
21 \\
42\end{array}$ & $\begin{array}{c}\text { Marignane } \\
\text { Marseille } \\
\text { Marseille } \\
\text { Saint-Fons (Lyon) } \\
\text { Lyon } \\
\text { Moulins } \\
\text { Lyon } \\
\text { Nice } \\
\text { Chambéry } \\
\text { Aninemasse } \\
\text { Lyon } \\
\text { Marseille } \\
\text { Lyon } \\
\text { Montpellier } \\
\text { Valence } \\
\text { Nimes } \\
\text { Marseille } \\
\text { Livron } \\
\text { Rognac } \\
\text { Livron } \\
\text { Le Teil } \\
\text { Grimaud } \\
\text { Beaune } \\
\text { Vercheny (Die) } \\
\text { Privas } \\
\text { Grenoble } \\
\text { Grenoble } \\
\text { Fos-sur-Mer } \\
\text { Saint-Jean-en-Royans } \\
\text { Le Bosquet d'Orb } \\
\text { Beauchastel (La Voulte) } \\
\text { Saint-Chamond } \\
\text { Riom } \\
\text { Livron } \\
\text { Valence } \\
\text { Livron }\end{array}$ & $\begin{array}{l}13 \\
13 \\
13 \\
69 \\
69 \\
03 \\
69 \\
06 \\
73 \\
74 \\
69 \\
13 \\
69 \\
34 \\
26 \\
30 \\
13 \\
26 \\
13 \\
26 \\
07 \\
83 \\
21 \\
26 \\
07 \\
38 \\
38 \\
13 \\
26 \\
34 \\
07 \\
42 \\
63 \\
26 \\
26 \\
26\end{array}$ \\
\hline
\end{tabular}

\section{RÉSULTATS}

Tournées [disponibilité sur route des véhicules 15 heures, tournée la plus longue (véhicule 8) 13 heures incluant le retour au dépôt]. 


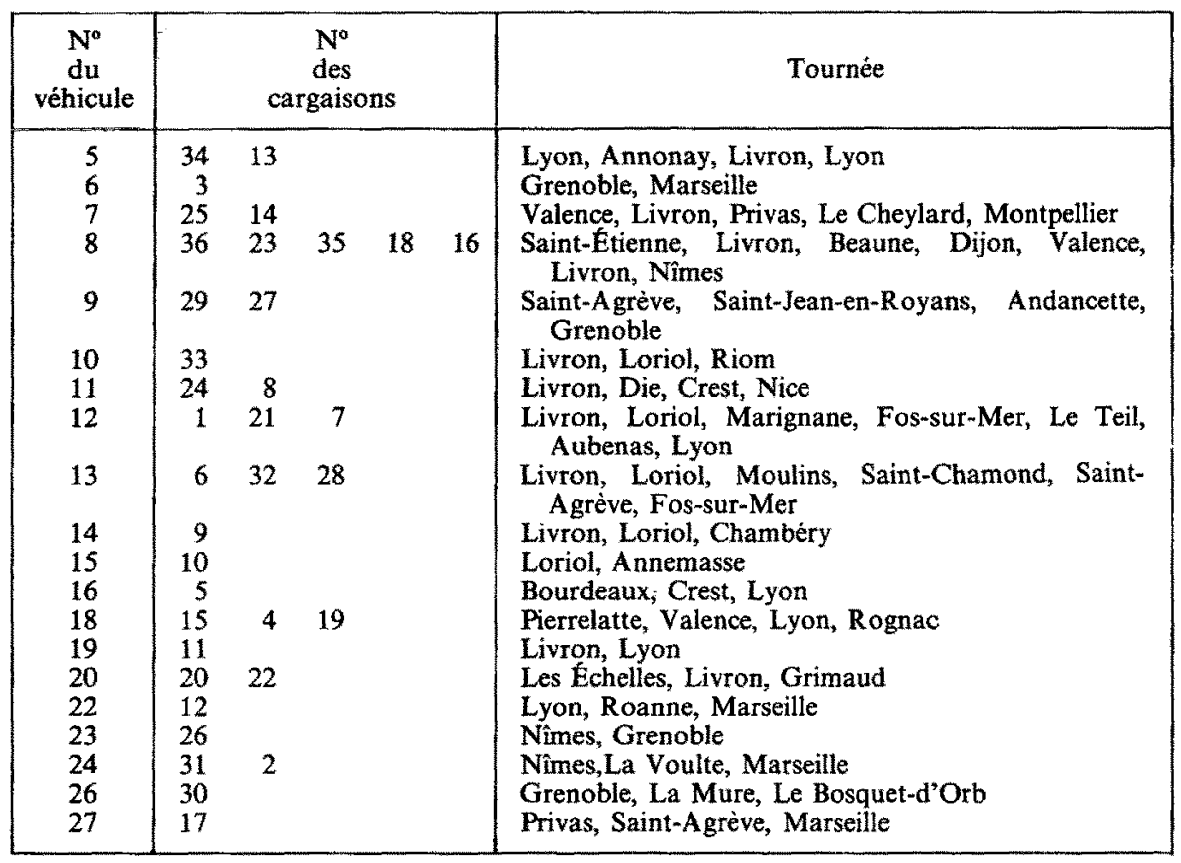

Tournées [disponibilité sur route des véhicules 9 heures, tournée la plus longue (véhicule 16) 9 heures incluant le retour au dépôt].

\begin{tabular}{|c|c|c|c|c|}
\hline $\begin{array}{c}\mathbf{N}^{\circ} \\
\text { du } \\
\text { véhicule }\end{array}$ & \multicolumn{3}{|c|}{$\begin{array}{c}\mathrm{N}^{\circ} \\
\text { des } \\
\text { cargaisons }\end{array}$} & Tournée \\
\hline 5 & 34 & \multicolumn{2}{|c|}{13} & Lyon, Annonay, Livron, Lyon \\
\hline 6 & 3 & & & Grenoble, Marseille \\
\hline 7 & 4 & \multicolumn{2}{|l|}{19} & Valence, Lyon, Rognac \\
\hline 8 & 36 & \multirow{2}{*}{\multicolumn{2}{|c|}{33}} & Saint-Etienne, Livron, Loriol, Riom \\
\hline 9 & 17 & & & Saint-Agrève, Marseille \\
\hline 10 & 11 & \multirow{2}{*}{\multicolumn{2}{|c|}{$\begin{array}{l}28 \\
22\end{array}$}} & Livron, Lyon, Saint-Agrève, Fos-sur-Mer \\
\hline 11 & 25 & & & Livron, Privas, Livron, Grimaud \\
\hline 12 & 24 & \multicolumn{2}{|c|}{5} & Livron, Die, Crest, Lyon \\
\hline 13 & 23 & \multicolumn{2}{|c|}{35} & Livron, Beaune, Dijon, Valence \\
\hline 14 & 1 & \multicolumn{2}{|c|}{21} & $\begin{array}{l}\text { Livron, Loriol, Marignane, Fos-sur-Mer, Le Teil, } \\
\text { Aubenas, Lyon }\end{array}$ \\
\hline 15 & 6 & 32 & 29 & $\begin{array}{l}\text { Loriol, Moulins, Saint-Chamond, Saint Agreve, Saint- } \\
\text { Jean-en-Royans }\end{array}$ \\
\hline 16 & 8 & & & Bourdeaux, Crest, Nice \\
\hline 18 & 15 & 18 & 10 & Pierrelatte, Valence, Livron, Loriol, Annemasse \\
\hline 19 & 9 & & & Livron, Loriol, Chambéry \\
\hline 20 & 20 & 16 & & Les Echelles, Livron, Nîmes \\
\hline 22 & 12 & & & Lyon, Roanne, Marseille \\
\hline 23 & 26 & 27 & & Nîmes, Grenoble, Andancette, Grenoble \\
\hline 24 & 31 & 2 & & Nîmes, La Voulte, Marseille \\
\hline 26 & 30 & & & Grenoble, La Mure, Le Bousquet-d'Orb \\
\hline 27 & 14 & & & Privas, Le Cheylard, Montpellier \\
\hline
\end{tabular}

vol. $18, n^{\circ} 4$, novembre 1984 


\section{BIBLIOGRAPHIE}

1. D. J. AgEE, K. P. STARK, C. R. MURRY et X. M. DEMouln, A Routing and Scheduling Problem for a Rail System: A Case Siudy, J. Opl. Res. Soc, vol. 32, 1981, p. $767-774$.

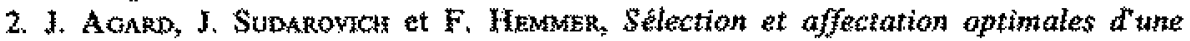
flote dations, R.A.I.R.O.Rech. Op., vol, 7, n 2, mai 1973, p. 3-26.

3. S. R. Axkotexar, N. R. PATEL et I. L. Sath, Optimization of Fehicte Schedulas for a Road Transport Corporation, Scientifte Mandsement of Transport Systems, N. K. JAEStAL, td. North Holland, Amsterdam, 1981, p. 244-253.

4. G. M. Buxer, The Ventcle Schedaling Problem and Monte Carlo simulation, 3 . Opl. Res. Soc, wol. 30,1979 , p. $563-573$.

5. T. B. Boffex, D. D. EDMond, A. 1. Hinxaman et C. J. Pursolove, Tow Approaches to Scheduling Container Ships with on Application to the North Atlantic Route, $\mathrm{I}$. Opl. Res. Soc, vol. $30, \mathrm{n}^{\circ} 5,1979$, p. $41342 \mathrm{~S}$.

6 5. M. P. Bollew, The Solution of a Railway Loconotive Scheduling Problem, $y$. Opl. Res. Soc., vol. 31. 1980, p. 943-948.

7. J. M. D. CANeoo et O. M. M. ExCALANTE, A Network Solution to a General Vehtcle Scheduling Problem, European Iournal of Operational Reseatch, vol 1 , 1977, p. 255-261.

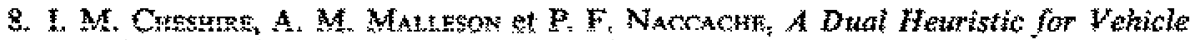
Scheduling, J. Opl. Res, Soc, vol. 33, 1982, p. $51-61$.

9. N. CHRtsromes, The Venicle Routing Problem, R.A.I.R.O.-Rech. Op., vol. 10, $n^{\circ} 2$, fêvrier 1976, p. $\$ \$-70$.

10. G. CEARKe et J. W. WaGHT, Scheduling of Vehicles from a Central Depot to a Number of Delitery Points, Operations Research, vol. 12,1964, p. $568-581$.

11. J. Desrosiers, Folms et M. Desrochers, Routing with Time Windows: Synthesis, 6 th European Congress on Operations Research, Vienne (A utriche), 19-22 juillet 1983.

12. J. C. Drverpax, Modale at algorithme de communde d un systeme de transport en commun à la demande, Thèse de doctear ingénieut, Université des Sciences et Techniques de lille, fevtrer 1974.

13. J. C. Drverraz el J. M. Toulotte, Modele de bus a la demande, R.A.M.R.O-Rech. Op, vol. $6, z^{\circ} 3$, octobre $197 \%$, p. 47+60.

14. M. Gondran at M. Moux, Graphes et Algorithmes, Eyrolles, 1979.

15. A. GUiNer, Le systeme T.I.R., wa systeme détablissement de Tournete Industrielles Routières, These de $3^{*}$ cych, Universite Claude-Bermard-Lyon 1 , jatwer 1984.


d'optimisation appliqués aux systeme de transport, résumé du Séninaire organiser par 1'1.F.A.C a Viente (Autriche) en février 1976, Automatisme, décembre 1976, p. $398-403$.

17. E. Hruxcos, Conception dun systeme interactif a atade d la conception des horatres pour Fexplotation du metro (SACHEM), Informatique et Gestion, $\mathrm{n}^{\mathrm{*}}$ 101, novenbre 1978, p. 60.64 .

18. Institat de Recherche des Transports, J. Fonturt, Programme tounées, Organtsathon de tournés de ramassage, 94110 Arcueil, avril 1974 .

19. Institut de Recherche des Transports, G. CAplain: Le probleme des townées: recherche dine amelioration des méthodes de résolution existantes, 94110 . Arcueit, 1977. 
20. H. W. KuHN, The Hungarian Method for the Assignment Problem, Naval Research Logistics Quaterly, vol. 2, $\mathrm{n}^{\circ} 1-2$, mars-juin 1955, p. 83-98.

21. J. P. Legendre et M. Minoux, Une application de la notion de dualité en programmation en nombres entiers : sélection et affectation d'une flotte d'avions, R.A.I.R.O.Rech. Op., vol. 11, n 2, mai 1977, p. 201-222.

22. R. E. Marsten et M. R. Muller, A Mixed-Integer Programming Approach to Air Cargo Fleet Planning, Management Science, vol. 26, $\mathrm{n}^{\circ} 11$, novembre 1980 , p. 1096-1107.

23. R. Mercatanti et L. SPanedda, La Recherche de l'ensemble optimal des itinéraires des véhicules dans une entreprise de transports automobiles extra-urbains, R.A.I.R.O.-Rech. Op., vol. 9, $\mathrm{n}^{\circ}$ 1, janvier 1975, p. 59-75.

24. G. Monty, Automatisation de Paffectation de locomotives, R.A.I.R.O.-Rech. Op., vol. $4, \mathrm{n}^{\circ} 1,1970$, p. 19-27.

25. J. M. PRoTh, Transport : une utilisation de l'outil informatique, Revue Française de Gestion, mars-avril 1981, p. 55-62.

26. R. Russel, An Assignment Routing Problem, Networks (U.S.), vol. 9, n 1, 1979, p. $1-17$.

27. C. D. T. WAtson-Gandy et L. R. Foulds, The Vehicle Scheduling Problem: $a$ Survey, New Zealand Operational Research, vol. 9, $\mathrm{n}^{\circ}$ 2, 1981, p. 73-92.

28. B. W. Wrlliams, Vehicle Scheduling: Proximity Priority Searching, J. Opl. Res. Soc., vol. 33, 1982, p. 961-966.

29. J. G. Weston, Operational Research in London Transport, J. Opl. Res. Soc., vol. $32, \mathrm{n}^{\circ} 8,1981$, p. $683-694$. 